\title{
Commoditization of PACS and the Opportunity for Disruptive Innovation
}

\author{
Bruce I. Reiner
}

Published online: 22 February 2013

(C) Society for Imaging Informatics in Medicine 2013

"When we all think alike, then no one is thinking." Walter Lippman

\section{Introduction}

The transition from film-based (i.e., analog) to filmless (i.e., digital) medical imaging represented a radical innovation in medical imaging practice and technology, which in large part was driven by the creation of the picture archival and communication system (PACS). For the first time, medical imaging professionals could capture, archive, transmit, and display medical images digitally, thereby eliminating the inefficiencies associated with hard copy film, which had been the norm for almost a century. This radical technology innovation in turn led to a myriad of medical imaging practice innovations including the outsourcing of professional medical imaging services (i.e., teleradiology), rapid report turnaround times (through speech recognition software), and instantaneous and ubiquitous access to medical imaging data. While the early adoption of PACS in the USA was in large part restricted to the academic practicing community, its dissemination over time has effectively permeated the entire medical imaging community. The result is that the PACS market has been transformed from a new purchaser to replacement market, which has dramatically reduced economic opportunity for PACS vendors.

Along with this shift in market dynamics, PACS technologic innovation has declined, which in part is attributable to

B. I. Reiner $(\bowtie)$

Department of Radiology, Veterans Affairs Maryland Healthcare

System, 10 North Greene Street,

Baltimore, MD 21201, USA

e-mail: breiner1@comcast.net its longevity. The early and mid-stages of the PACS S-curve were defined by incremental innovations leading to substantive performance gains in workflow, productivity, and operational efficiency, which effectively created a positive return on investment (ROI) for PACS vendors [1]. The late stage of the PACS S-curve has been associated with a relative dearth of innovation and lack of performance improvement measures, which has led to its commoditization. The resulting declines in PACS profit margins and new sales have in turn lead to a further decline in R\&D expenditures, which perpetuates this commoditization trend and lack of innovation. While this may seem like a lost economic and technologic cause, the reality is that these market conditions have created an opportunity for disruptive innovation. If successful, this could reinvigorate the PACS marketplace and produce a new cycle of technologic and practice innovation.

\section{The Principles of Disruptive Innovation}

Disruptive innovation is a concept developed by Bower and Christensen [2], which describes a process by which a product or service is established through a selectively simple application in a niche market, which then expands throughout the broader market to eventually displace established companies and their sustaining technologies. These sustaining technologies are the modus operandi of traditional markets and are created with the goal of incremental improvement, which in turn serves to foster continuous and predictable sales within the existing customer base. Disruptive technologies, on the other hand, represent new nontraditional products, which initially focus on an underserved market segment, which often cannot afford the cost of traditional sustaining technologies. While these disruptive technologies generally underperform the established 
products they compete against, they typically offer a unique value, thereby gaining a foothold in the underserved market segment. Characteristics of disruptive technologies in their initial stages may include lower gross margins, smaller target markets, and simpler products and services, which are all unattractive to traditional companies operating at higher tiers (i.e., more profitable) of the market. This lack of interest on the part of traditional vendors creates an opportunity for the disruptive technology producers to innovate and create new technologies in the relative absence of competition.

The five principles of disruptive technologies as described by Christensen are listed in Table 1 [3] and explain how traditional market economics and technology development creates the opportunity for disruptive innovation to evolve. In order to maximize profitability, traditional companies (who are beholden to their investors) focus their research and development resources on existing products and services (i.e., sustaining technologies), which are directly tied to the perceived needs of their existing customer base. If customer feedback indicates there is little interest in a proposed innovation, it will in all likelihood be squashed in its infancy. As a result of this myopic focus on the "here and now," an opportunity is created for nontraditional companies looking to radically innovate and exploit existing market deficiencies. Once a disruptive innovation has been successfully implemented, it is typically too late for the traditional company to follow suit, due to the significant first mover advantage associated with disruptive innovation $[4,5]$. The commonly employed strategy of traditional companies to delay innovation until the market is established and large enough to satisfy its financial needs is often its undoing. The one exception to this generalization in which established companies have been successful in disruptive innovation is when the traditional company creates an autonomous entity charged with creating a new and independent business, separate and distinct from the parent company and its risk-averse culture.

Companies whose investment processes demand quantification of market size and ROI often become paralyzed when faced with disruptive technologies because they demand market data which do not exist [6]. By the time the disruptive innovation has been commercialized and market data are available for analysis, the traditional company is too late to effectively impact and profit from the innovation opportunity. It is important to note that while disruptive

Table 1 The five principles of disruptive technologies

1. Companies depend on customers and investors for resources.

2. Small markets do not solve the growth needs of large companies.

3. Markets that do not exist cannot be analyzed.

4. Technology supply may not equal market demand.

5. An organization's capabilities define its disabilities. technologies customarily target small markets initially, they often become competitive in the broader market and erode the profitability of traditional sustaining technologies. This is particularly relevant to those sustaining technologies which are "long in the tooth" (i.e., mature) and have reached the plateau on their S-curve. The greatest performance gains associated with these technologies are in the past and new engineering efforts are apt to realize minimal performance gains. The alternative disruptive technology can effectively exploit these limitations by offering an entirely new and novel approach, often at a marked reduction in cost to its "traditional" competition.

The combination of existing commoditization pressures, declining reimbursements for medical imaging providers, and maturity of PACS technology have collectively have created a timely opportunity for disruptive innovation. The challenge is to identify an innovation strategy which provides both a more cost-effective and value-added alternative to traditional PACS.

\section{The Evolution of PACS Technology and Existing Deficiencies}

PACS technology has undergone fairly substantive change in architecture and functionality over the past two decades. In its infancy, PACS was designed to emulate a film view box, with current and prior images transferred from the modality and archive to the PACS workstation for review and/or interpretation. This model was required due to limitations of network speed and computer performance which made it impractical to have studies made available "on demand." It required a good deal of computer memory and processing power, with the major limitation being the need to anticipate and "prefetch" required historic images.

The next major advance in PACS functionality made images available for transfer on an "as needed" basis. This required a faster network and added tremendous flexibility for end users using the PACS to specify the images needed at the time of interpretation or review. This obviated the requirement to prefetch studies and dramatically increased image accessibility and workflow. A variant of this architecture is one that uses "on the fly" (i.e., on demand) image compression that can take an original dataset and transfer images to fit the resolution of a monitor in real time. Advanced workstation vendors have migrated toward the use of this type of server side rendering in which the graphical processing and image loading take place at a server which is remotely located.

With this architecture, the clients (i.e., workstations) have a software that opens up a window into the server and actions (e.g., window/level, zoom, rotate) requested at the workstation using a variety of input devices (e.g., mouse, trackball, game controller, stylus), performed on the server, 
and then displayed on the client workstation. The limitations of this architecture are that users currently are required to load software for each of the multiple applications used in diagnostic imaging even when using a server side rendering approach. This is due to the fact that each commercial image visualization and analysis system is optimized for a specific subset of tasks. Interactions among various graphical user interfaces may lead to software conflicts. Additionally, different applications may require different input or output devices. Consequently, end users must run multiple different software programs, often on multiple different computer workstations. This often becomes impractical for everyday use in a busy working environment, and as a result, many radiologist end users opt to limit the number of applications in use, which has the potential to adversely affect outcome (e.g., diagnostic accuracy).

Vendors within the medical imaging and information system sectors have held onto this concept of proprietary PACS workstations because it creates the mentality of "one size fits all" and creates a dependence of the end users on a single vendor. While many technology users prefer a "best of breed" approach, this has to date not been practical when it comes to the myriad of applications intrinsic to PACS. The multiple steps that occur in the imaging chain (image acquisition, archival, transmission, display, processing, interpretation, and reporting) are intrinsically tied to a single PACS, thereby requiring the end user to adapt to a single vendor's technology. The alternative, which is often practiced, is to have multiple stand-alone workstations, each performing a single set of functions. For example, a radiologist who wants to simultaneously review nuclear medicine and MRI exams on a single patient is often tasked with migrating between several different workstations: one for review of the nuclear medicine (e.g., PET/CT exam), one for the MRI exam, and another for advanced (e.g., 2- or 3-D multi-planar image) processing. If that same radiologist wants to extract clinical data associated with the patient in question, he/she needs to search a separate workstation tied to the patient electronic medical record (EMR). For reporting (using a structured reporting application), the radiologist may be required to access a separate computer workstation that may or may not have an integrated critical results reporting package. If that is not enough, the radiologist may want to utilize computerized decision support applications during the interpretation process, requiring separate software applications and potentially an additional computer workstation. While some of these applications may be "integrated" into the central PACS architecture, the integration is limited in scope and functionality. In reality, it requires opening up a separate application from the main PACS workflow engine, which takes time and memory. The solution to this is a single, fully integrated workstation which provides transparent and instantaneous access to a variety of software programs, each of which is fully integrated into a single workflow engine.

\section{PACS Portal: a Disruptive Innovation Strategy}

The proposed innovation strives to address the technical, operational, workflow, and economic deficiencies intrinsic to existing PACS technologies. While traditional PACS vendors have a strong (and self-serving) interest in maintaining the existing model, it is important to realize that existing commoditization trends and maturity of the technology will effectively stifle any substantive future innovation. As a result, the only viable alternative is to seek out disruptive or transformative innovation strategies which transform the existing "single vendor" PACS technology model into a "multi-vendor" model which encourages a best of breed approach to technology selection, functionality, and performance. For simplistic purposes, we will refer to this proposed innovation strategy as a "PACS Portal"; although this innovation strategy does not need to be restricted to radiology alone and could be readily generalizable to all healthcare disciplines.

The PACS Portal serves as a "meta-worklist" which can span across multiple information system technologies (e.g., PACS, RIS, HIS, EMR). The system is able to "listen for" or retrieve HL7 (or other standards based) messages and create a list of imaging studies (or other healthcare studies or procedures in the case of a non-imaging portal). The radiologist can then create a filter which can display or manipulate a subset of the list of all studies or procedures performed and create an interpretation worklist from this. A clinician can create a list of his/her patients, tumor board cases, or another type of subset of the total number of procedures as well. The portal can in turn automatically bring up the appropriate context-specific viewer for the defined study. For example, a $\mathrm{CT}$ of the abdomen and comparison study might be viewed on a generic 3-D cross-sectional server side display program while an ultrasound study of the heart might be viewed using a cardiology echocardiography display and quantitative analysis program.

The PACS Portal takes a given study on the worklist and then uses group- or user-defined protocols to determine the appropriate software to view the study. The images are then retrieved from a central storage device or a distributed one and sent to the appropriate server or servers for interactive rendering, image analysis, and image optimization. When multiple programs are available for a given single study or combination of studies, then the system can either use preselected server side rendering programs or alternatively can allow the user the option of selecting the ones most appropriate for use. The system has the capability of obtaining feedback from 
the users as to whether the software was appropriate for the intended use (i.e., satisfaction score) and utilize this user and context-specific data for future reference and application selection.

A number of theoretical advantages can be gained through the creation and implementation of a PACS Portal. The first one is the ability to take advantage of server side rendering technology at the portal. This allows image processing and analysis to begin immediately at the server or using multiple servers (even more efficiently) without having to wait for the image or patient information to be transferred in its entirety to the workstation. The servers can be set up with a much faster connection than would be practical for each workstation and consequently the workstation represents a window into the memory of the server or servers which can be opened much more quickly without the constraint of waiting for an entire image or information dataset to be transferred. The raw image data do not need to be sent to the interpreting radiologists or clinicians (which can take a good deal of time) but rather the results of the image processing and image rendering engine can be sent and tailored to the individual workstation. Secondly, the system would allow individual display and image processing and enhancement vendors to create their own hardware and software solutions using technologies such as clustering or grid computing to optimize performance. The only requirement would be that these vendors would adopt the standard of the PACS Portal for accessing the software package. The portal would define "standards or conventions" which could be used to make the user interface to various software packages more uniform and thus easier to use. A third (and perhaps most important) advantage of this approach would be that individual end users could pick their own best of breed solution to individual software applications, which could include image display, processing, analysis, decision support, reporting, and communication. With continued use of the PACS Portal model, the database could be queried to automate software application selection, based upon each individual end users' usage and feedback. At the same time, meta-analysis of other end users with similar profiles could be performed to assist in providing additional guidance to software selection, with the goals of improving productivity, workflow, and interpretation accuracy [7].

The PACS Portal model could in theory dramatically change the economics of PACS, from the combined perspectives of vendors and end users. Software vendors would now have a strong economic incentive to invest in research and development, with the hopes of increasing usage and market share of their products. This could also lead to the creation of entrepreneurial small companies to create and refine additional software applications not currently available in the single all inclusive PACS model. The traditional
PACS vendors would be faced with heightened competition from these single application vendors and in turn be challenged to accelerate their own innovation efforts in order to maintain viability in the marketplace. In short, the forces of natural selection will be at play and empower end users to select vendors based upon value, as opposed to the captive audience the current market mandates. The manner in which these software services are paid for can also undergo transformation. Instead of the current model of up-front all inclusive pricing, vendors may opt to provide customers with a number of pricing options including (but not limited to) fee for use, individual licensing, and bundling of software packages. It would not be beyond reason to have individual software providers offering periodic pricing specials to attract new users (i.e., the daily K-mart blue plate special), thereby stimulating competition. In the end, added-value products will prosper, which should be the goal of any quality-centric service or industry.

In a bit of irony, the data derived from this disruptive innovation could in turn stimulate future innovation. Vendors would have the ability to determine comparative usage and end user satisfaction of their technologies relative to competitors. In addition, if electronic auditing tool functionality were to be integrated into the PACS Portal [8], vital workflow data would be collected to identify workflow inefficiencies that can be targeted for future technology development and refinement. The goal is to create technology that is iterative in nature, constantly evolving and refining itself to improve performance and end user satisfaction. The ultimate goal is to reinvent PACS as we know it, and the only logical way to do so is through disruptive innovation.

\section{References}

1. Siegel EL, Reiner BI, Kuzmak PM: Adoption of PACS by the Department of Veterans Affairs: the past, the present, and future plans. Proc. SPIE 3980, Med Imaging 2000: PACS Des Eval Eng Clin Issues 3980:134-138, 2000

2. Bower J, Christensen CM: Disruptive technologies: catching the wave. Harv Bus Rev 73:43-53, 1995

3. Christensen Clayton M: The Innovators' Dilemma: When New Technologies Cause Great Firms to Fail. Harvard Business School Press, Boston, 1997

4. Markides C: Disruptive innovation: in need of better theory. J Prod Innov Manag 23:19-25, 2006

5. Pisano G: Profiting from innovation and the intellectual property revolution. Res Policy 35:1122-1130, 2006

6. Reiner BI, McKinley M: Innovation economics and medical imaging. J Digit Imaging 3:325-329, 2012

7. Reiner B: One size (doesn't) fit all. J Am Coll Radiol 4:567-570, 2008

8. Reiner B: Automating radiologist workflow. Part I: the digital consultation. J Am Coll Radiol 10:1080-1085, 2008 